\title{
Experimental studies on Bio-Home Automation using loT aiming for Security and Safety
}

\author{
Venkata Sumana C H'1 Dr. Latha B M and Manjula B K \\ Department of Electronics and Communication EngineeringGM Institute of Technology Davangere India
}

\begin{abstract}
An emerging technology that makes the world smarter is the Internet of Things (IoT). The growth of the IoT network has enriched the home and lifestyle with its associated technologies. Life today revolves around the notion of automation and the items that are automated are said to be of the next generation because they decrease human intervention. The technology of the home automation system is unique to other systems that allow the user to control the system through an internet connection from any location around the world. The proposed work reflects the many IoT applications that use Raspberry pi, sensors, IoT module to design the smart home automation and home security monitoring framework. The smart home automation system uses an android smartphone to easily control home appliances from any location around the world through an internet connection and will act as a smart monitoring security module for home security monitoring. Traditional surveillance systems only record motion-based movements, but the proposed system serves the function of facial recognition to minimize the error caused by motion detection, and since it records automatically only during motion detection, the system saves a lot of storage space. Once the motion is detected by the PIR Sensor and the image is sent to the Android app via Raspberry pi, the Raspberry pi camera module is used to capture photos. This device can track when motion is detected and scans for the faces caught in the picture and operates with the aid of the door for face recognition. The key benefit is that the owner can seek surveillance from any part of the globe and can take action according to the circumstances.
\end{abstract}

KEY WORDS: IOT; RASPBERRY PI; FACE RECOGNITION; PIR SENSOR; ANDROID.

\section{INTRODUCTION}

Nowadays, in technology where everything is automatically run, progress is too rapid. It is a remarkable idea to include the latest upgraded equipment in the construction of a home automation system with safety measures. A smart home device (Casado-Vara 2019; Guo et al. 2018; Abdel-Basset et al. 2019) can be built using the Internet of Things (IoT). In order to build a good system focused on the communication of these objects, the IoT can be

\section{ARTICLE INFORMATION}

*Corresponding Author: sumanavch@gmit.ac.in

Received 7th Oct 2020 Accepted after revision 28th Dec 2020

Print ISSN: 0974-6455 Online ISSN: 2321-4007 CODEN: BBRCBA

Thomson Reuters ISI Web of Science Clarivate Analytics USA and Crossref Indexed Journal

\section{Clarivate
Analytics}

NAAS Journal Score 2020 (4.31)

A Society of Science and Nature Publication,

Bhopal India 2020. All rights reserved.

Online Contents Available at: http//www.bbrc.in/

Doi: $h$ ttp://dx.doi.org/10.21786/bbrc/13.13/53 referred to as a network of physical things interconnected together in the form of electronics, software, sensors and communication networks(Hasan et al. 2019; $\mathrm{Xu}$ et al. 2019). In different fields, such as finance, military or personal security industries, surveillance is an important aspect(Gandhi et al. 2018). Surveillance systems are proving to be a significant source of defense due to the exponential increase in burglary and theft operations(Gandhi 2018). Individuals rely on advanced technologies for their security purposes because of ever growing technology. Due to their cost-effective design and easy maintenance(Khan et al. 2020).

security systems such as CCTV have proven to be extremely common for security purposes. For law enforcement, surveillance is very useful for investigating/ preventing illegal activity, identifying and tracking threats. Monitoring systems have also often played a crucial role in coping with breakdown cases(Shin et al. 2019; Dautov et al. 2018). Such CCTV devices aim to

$$
344
$$


constantly track activities. This results in high use of resources and waste of memory. In addition, it does not provide notice of any detected suspicious activities. Other than CCTV systems such as Retina scanners, fingerprint scanners, IR lasers, RFID systems are available only with the disadvantage that they are cost-effective with high deployment and maintenance costs. Hence, such systems are not a preferred way for security purposes for small scale applications. The proposed framework covers all these disadvantages through its reliability and portability. This method of surveillance is low-cost and also user-friendly.

Motivation: There is a rapid increase in the use of new technologies in the IoT environment. A smart home is one of the IoT applications as well. There are several problems emerging from exponential growth in technology and changes in architecture that include how to handle and monitor the whole system, security in smart homes. In most situations, the question of home protection relies on the physical presence of a person in the home and in commercial buildings. Electricity has become one of the key contributors to energy usage, so it is important to minimize this energy consumption. The main aim of this work is to build a cost-effective control system for home appliances, to find it difficult to control home appliances from a distance, and to provide comprehensive home protection and safety measures. The proposed system is a home automation and security system based on IoT that aims at developing a reliable system to provide a reliable home automation solution.

Not only does the smart word minimize efforts, but it also lowers power investment. Smart homes can be briefed in order to provide their residents with convenience, quality, protection and energy efficiency as the integration of technology within the home environment. Intelligence in home systems will essentially boost the quality of life of the elderly and disabled. IoT makes it possible to connect from anywhere used in residential needs that are of electrical and technological origin. Monitoring the home's gadgets when necessary is one way to monitor the amount of energy used. At our convenience of time and location, the added intelligence such as Raspberry Pi lets the home appliance work and power. The Raspberry $\mathrm{Pi}$ is responsible for controlling electrical appliances and providing users with authentication and protection. The proposed Raspberry Pi face recognition protection system that can be linked to the smart home system and the system saves a lot of storage space because it records only during motion detections automatically.

\section{Objectives}

- The main objective is to design and execute a cost effective and open source home automation and surveillance system.

- To control the home appliances conveniently from any location around the world through an internet connection by using an android smart phone.

- To provide security system for smart home automation using Raspberry Pi in terms of face recognisation.
- To overcome the shortcomings of home security systems by providing information of current situation when the owner is away from the house.

- To make lifestyle comfortable and easier way such as to reduce the power consumption, to do work effectively and timely manner and to help the physically challenged people.

Literature Survey: (Patchava et al. 2015) It offers an automation system in which home appliances are operated using the internet through a laptop or mobile device, which in turn eliminates most human interactions by supporting the Internet of Things system. For sensing and tracking purposes, the Raspberry Pi operates and monitors motion sensors and video cameras. For example, it captures the identity of the intruder and uses basic computer vision technology to detect its presence (CVT). The cameras begin recording whenever motion is detected, and the Raspberry Pi system alerts the owner via an SMS and an alarm call.

(ShariqSuhai et al. 2016)A multi-functional safe smart home (SSH) system was developed. It is a security framework based on GSM that allows simple communication between the pre-configured number and the system. The consumer will receive warnings anywhere in the world because GSM is a wireless technology, thereby making the device independent of location. For example, if motion is detected in any room when the system is activated, when the system is activated and de-activated for fire detection and smoke detection within a certain range, the owner is alerted by an SMS. When motion is observed, GSM technology is often used to call the owner.

(Quadri \&Sathish 2017) Different Linux, Python, IoT, HTML and Raspberry Pi concepts have been implemented here to effectively implement the framework. In order to allow only approved users to access, a website is provided with a username and password for the end user. Using open and close buttons, the user can monitor the door after successful login and watch the live streaming video of the desired location, i.e. the proximity of the door. In several areas, such as banks, hospitals, laboratories, offices, etc., the device can be used to significantly reduce the danger of unauthorized entry.

(Wati et al. 2017) This study proposed a smart home protection face detection and recognition system that captures images and performs image processing using MYRIO 1900, which is the main controller containing image acquisition, face detection and face recognition software. The user interface, image display and monitoring of the personal computer (PC) are used. MYRIO and PC are both programmed using LabVIEW, which is called ' $G$ ' as a graphical programming language. Compared to the raspberry $\mathrm{Pi}$, the big drawback here is that MYRIO's cost is very large.

(Pawar et al. 2018) An ideal smart home security solution with less expense and improved security has been proposed and demonstrated by the author. With the 
aid of IoT and Face Recognition, advanced protection levels are given. The Web camera is attached to the Raspberry Pi with sensors such as Passive Infrared (PIR) and Ultrasonic sensors. The camera captures a picture of the person in front of the door on motion detection, then real-time face recognition is performed using local binary patterns (LBP). If the picture of the individual matches one of the members of the household, then the door will open, otherwise the doorbell will ring. On this paper, the proposed work is based.

(Medapati et al. 2020) applied artificial intelligent method in IoT detection system to predict and match the face from the database. The Perona-Malik diffusion algorithm is applied in facial images captured from IoT sensor. The face location is cropped from the image and geometric face shape model is created. The Fisher linear discriminant analysis is applied to extract the different facial features from face model. The Convolutional network is used to train the derived features. The retrieval neural network and adaboost large memory usage is applied for face recognition process.

\section{METHODOLOGY}

A. Project description: This module describes an idea of controlling home appliance through IoT as well as motion based face recognition, which enables the home Security. Also the owner can control the home appliances by himself through IoT. It has mainly 3 parts: WebIOPi, PIR, and Camera.

WebIOPi: Here, webioPi software is used to control home appliances, and created a personal website to control the things like fan, TV, and light etc. HTML code is written to create website, CSS and JavaScript to create buttons and linking with RPi. Using above scripts created buttons to control home appliances.

PIR: PIR sensor is used to detect motion within certain distance. With the help of PIR sensor, RPi will knowing that there is a person in front of door, then automatically it turns on camera.

Figure 3.1: Block diagram of the proposed model

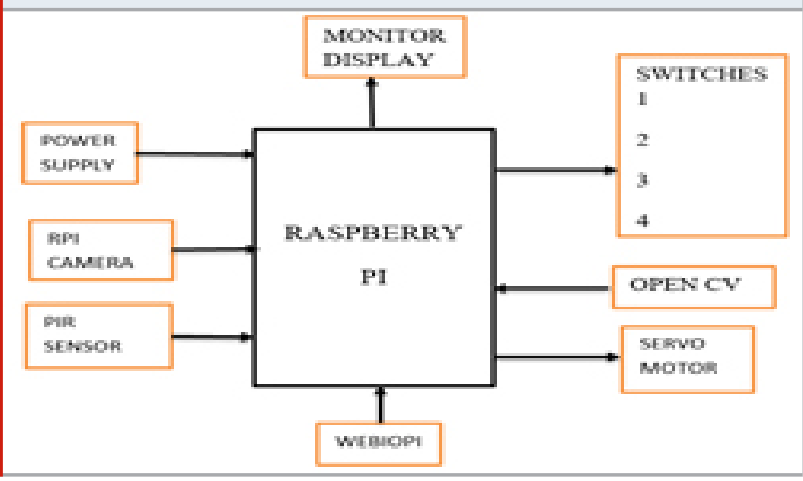

Camera: Here camera will open, based on motion detection using PIR sensor. Camera will start recognizing the face based on frontal face algorithm using open CV. If person is authorized then it will opens the door. If it detected as an unauthorized person then it will capture image of person and send to the owner mail.

Figure 3.2: Circuit diagram of the proposed model

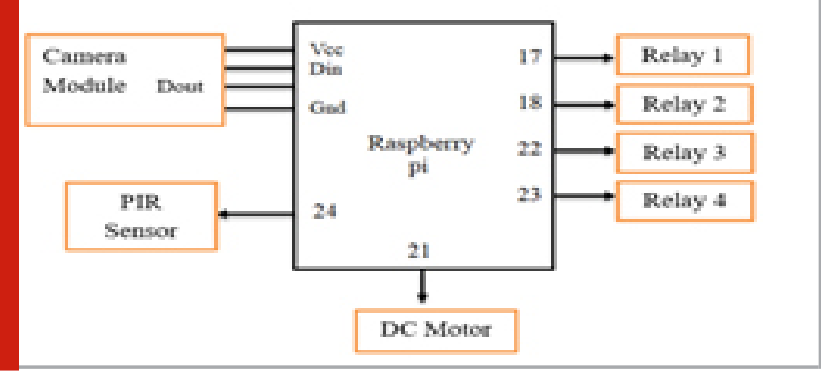

Figure 3.3: Web Controlled Home Appliances Flow Chart

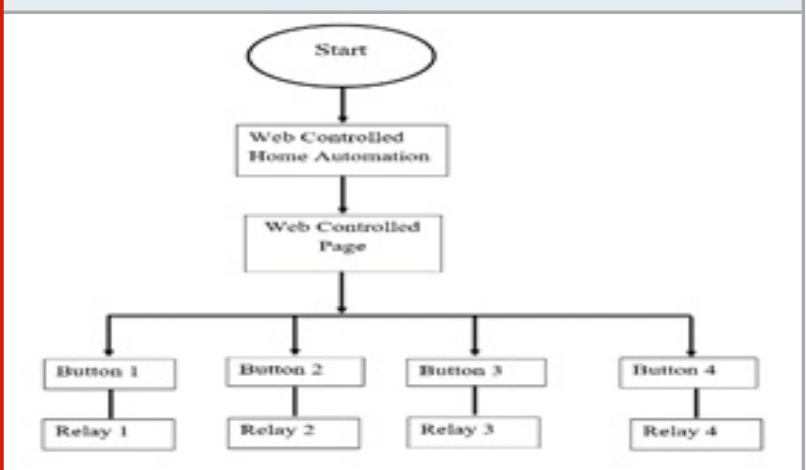

Figure 3.4: Motion based door open flow chart

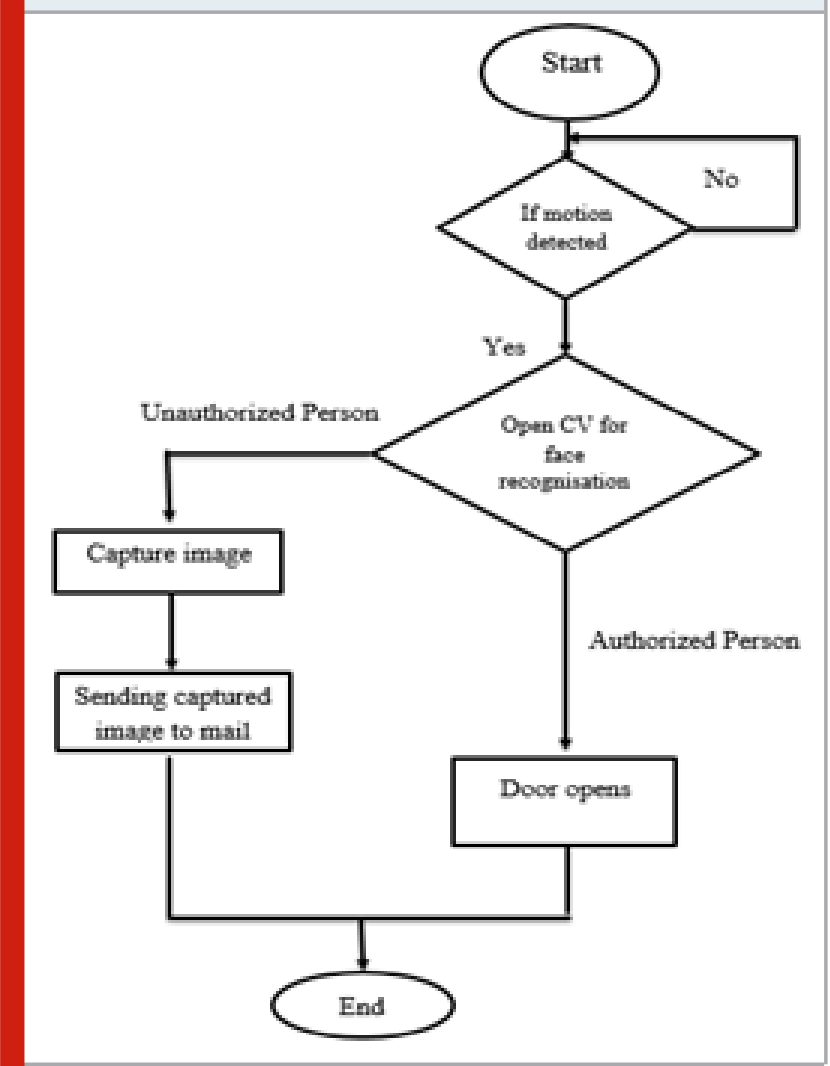


B. Open CV: Open CV is the massive open-source library for computer vision, machine learning, and image processing, and it now plays an important role in realtime activity in today's systems. By using it, it is possible to process photographs to classify human objects, faces, or even handwriting.Python is able to process the Open $\mathrm{CV}$ array structure for analysis when it is combined with different libraries, such as Numpuy. It utilizes vector space and performs mathematical operations on these features to classify image patterns and their different characteristics.

\section{OpenCV Functionality:}

- Image/video I/0, processing, display (core, imgproc, highgui)

- Object/feature detection (objdetect, features2d, nonfree)

- Geometry-based monocular or stereo computer vision (calib3d, stitching, videostab)

- Computational photography (photo, video, superres)

- Machine learning \&t clustering (ml, flann)

- CUDA acceleration (gpu)

WebIOPi: WebIOPi Controls, debug, and use Pi’s GPIO, sensors and converters from a web browser or any app. WebIOPi is the perfect Swiss-knife to make connected things. Developed and provided by Eric PTAK (trouch) Runs on Raspberry Pi.

Figure 3.5: Web Controlled Home Appliances

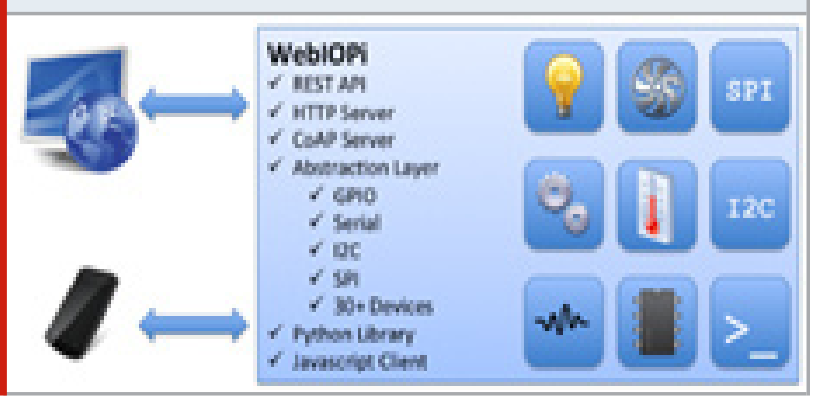

Html code is written to create website, css and JavaScript to create buttons and linking with RPi. Using above scripts created a buttons to control home appliances. If everything works properly, a web page is linked to different sections. Click on the GPIO Header link to switch to a simple web app for controlling the GPIO pins. WebIOPi software is used to control home appliances, and created a personal website to control the things like fan, TV, and light etc.

Once WebIOPi is up and running, Can point browser to http://yourraspberryIP:8000 and $\log$ in using the webIOPi username and the raspberry password.

To run web controlled program issue following: sudo /etc/init.d/webiopi start username:webiopi pw:raspberry
Then open browser and type rpi ip address like 192.168.43.39:8000, then hit enter web page will be opened then can control the appliances.

2. To stop program enter

sudo /etc/init.d/webiopi stop

Figure 3.6: Login in with default credentials
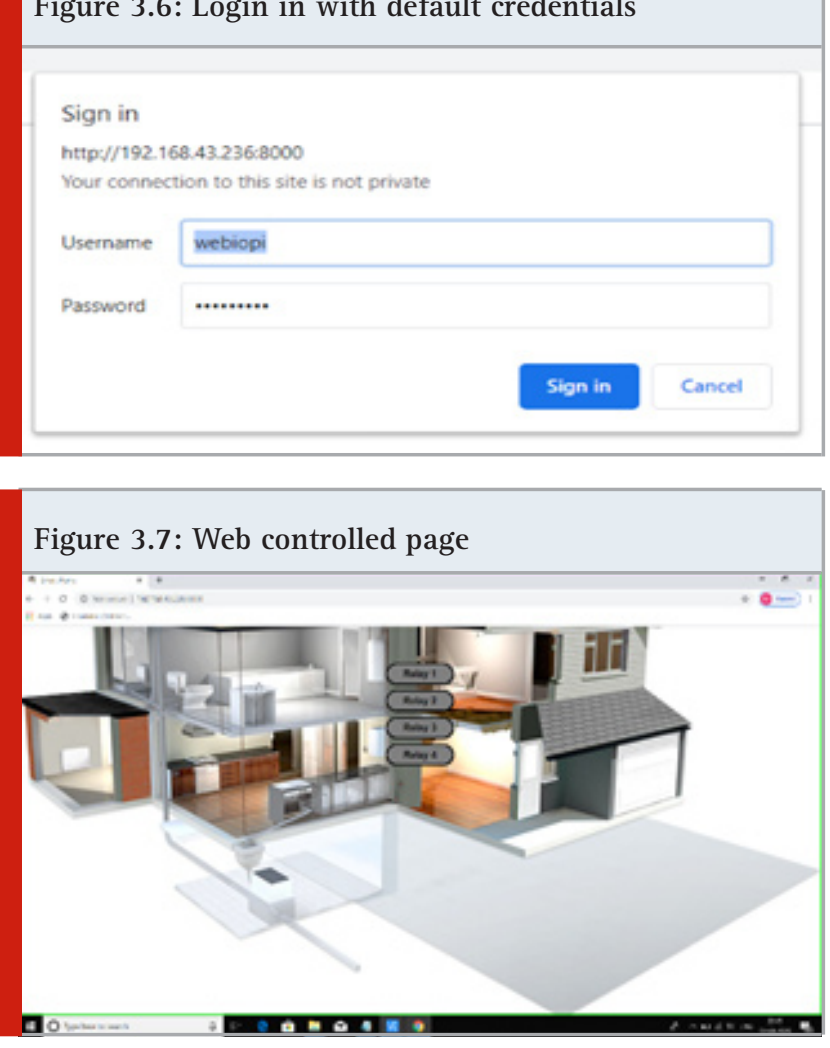

D. Face Recognisation Surveillance System: The System Works on 3.3V and 5V DC supply. Components connected to Raspberry pi-3 are PIR sensor, relay driver circuit, camera module, pen drive USB. From the Raspberry pi, by means of wifi configuration and IoT can control home appliances using sensors by means of relays. Relay are the switches that open or close circuits electronically. Here going to get email alert about the condition of the sensors. Already fixed one G-mail in Raspberry pi, SMPT protocol taking user name and password with that gmail and sending to our predefined mail ID's. PIR Sensor is used to detect the motion of the person, once motion is detected; camera opens automatically and checks for the authorised person face for the given time. If person face is matched with the previously stored datasets with unique ids then the door opens. The door only opens after a successful recognization process, when the face is stored previously in the database and that image has got the access to open the door then it will work otherwise it won't open. Otherwise camera captures the image of a person and sends it to the mentioned Email Id.

Face Recognisation consists of three phases ,they are:

1. Face detection and data collection.

2. Training recognizer.

3. Facial recognition. 
1. Data Gathering for Face Detection: The first task is to collect the data for which the classifier will be educated. It will write a python code that uses the OpenCV pretrained classifier to take 30 faces of each entity. OpenCV also provides several pre-trained face, eye, smile, etc. classifiers.Capture several faces from multiple users to be stored in a database, Faces will be stored in a directory: dataset, each face will have a specific integer numeric ID such as 1, 2, 3, etc.

2. Training the Recognizer: The recognizer is now trained according to the data collected in the previous phase. The LBPH face recognizer, included in the OpenCV kit, will be used. Retrieve the current working directory path and transfer to the directory where the picture directories are stored. Then switch into each directory of images and search for the images. Convert it to the NumPy array if the image is present. After that, perform the face detection again to make sure to have the correct images and then prepare the training details. Store the dictionary that includes the label IDs and directory names. Explore the data and save the file now. This code produces a file called trainer.yml and marks the files that the recognition code uses.Training Multiple Faces stored on a Database:

Each face should have a unique numeric integer ID as 1,2 , 3, etc

LBPH computed model will be saved on trainer/ directory.

PIL installs with pillow library with "pip install pillow"

3. Use the Facial Recognition Recognizer: You can now use the recognizer set up in the previous section to identify faces. If the face matches, the door opens using the Servo Motor.

\section{RESULTS AND DISCUSSION}

The main objective of the proposed work here is to create a system where it will be easy to operate home appliances and equipments very easily by making this system user friendly. Developing a smart home system was not easy at first. The most important part of this proposed work is human surveillance which is important due to the security issues of smart homes. For surveillance using face detection and face recognization is being used which are the most modern form of surveillance. PIR Sensor detects the motion, once motion is detected; camera opens automatically and checks for the authorised person face for the given time. If person face is matched with the previously stored datasets with unique ids then the door opens.

The door only opens after a successful recognization process, when the face is stored previously in the database and that image has got the access to open the door then it will work otherwise it won't open. Otherwise camera captures the image of a person and sends it to the mentioned or desired Email Id.
For this purpose Raspberry $\mathrm{Pi}$ is being used camera and OpenCV which is a open source which is a part of Python language. Python here acts as the main platform where most of the work is going to be done. Image processing needs to be done for the Face recognition. OpenCV is a library of open source computer vision applications. The library has a lot of streamlined algorithms, including face detection and recognition, which can be used in many IoT-related sectors. As this project's libraries used the HAAR classifier, the LBPH face recognizer was used.

Figure 4.1: Side view of the project setup

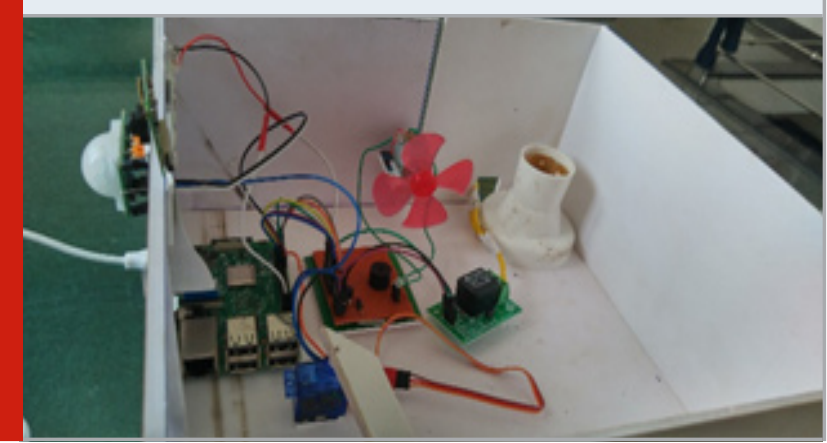

Figure 4.2: Front view of the project setup

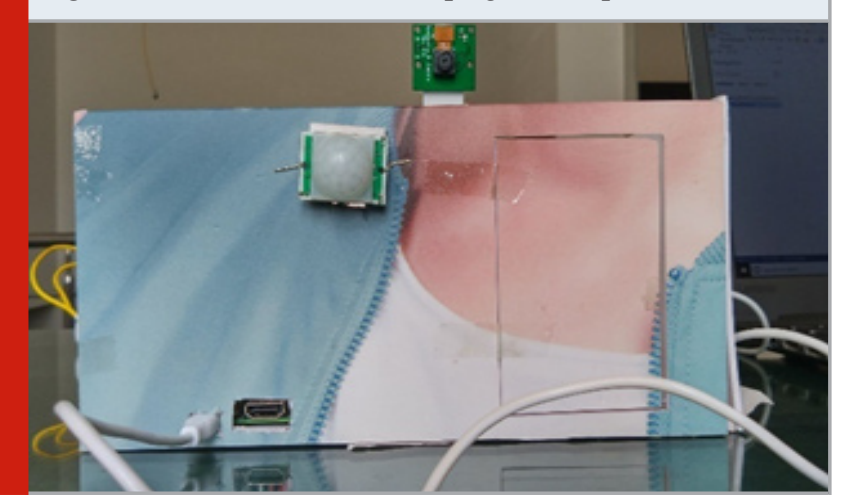

a) Web Controlled Home Appliances: WebIOPi software is used to control home appliances, and created a personal website to control the things like fan, TV, and light etc. HTML code is written to create website, css and JavaScript to create buttons and linking with RPi. Using above scripts created a buttons to control home appliances. It includes

1. Light is controlled through relay 2 button from web page.

b) Face Recognisation: PIR sensor detects motion detection, once motion is detected; camera opens automatically and checks for the authorised person face for the given time. If person face is matched with the previously stored datasets with unique ids then the door opens.

The door only opens after a successful recognization process, when the face is stored previously in the database and that image has got the access to open the door then it will work otherwise it won't open. Otherwise 
camera captures the image of a person and sends it to the mentioned or desired Email Id.

1. PIR sensor detects Motion and camera opens automatically.

2. Authorized person with matching Id

3. Door opens

4. Unauthorized person

Figure 4.3: Light is controlled by relay button 2 from web page
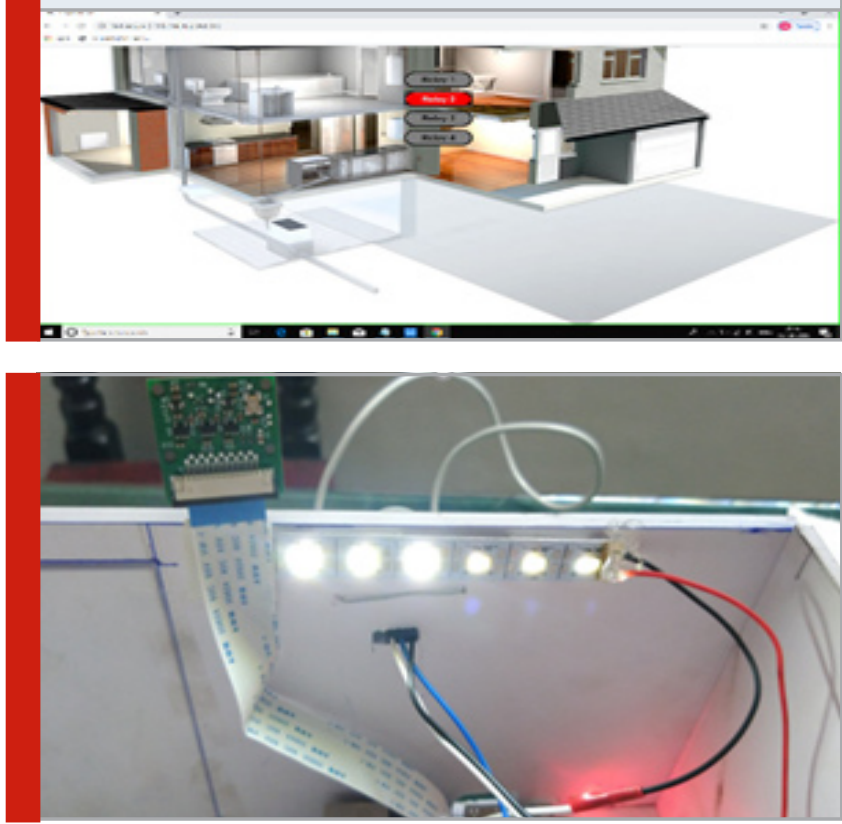

Figure 4.4: Fan is controlled by relay button 3 from web page

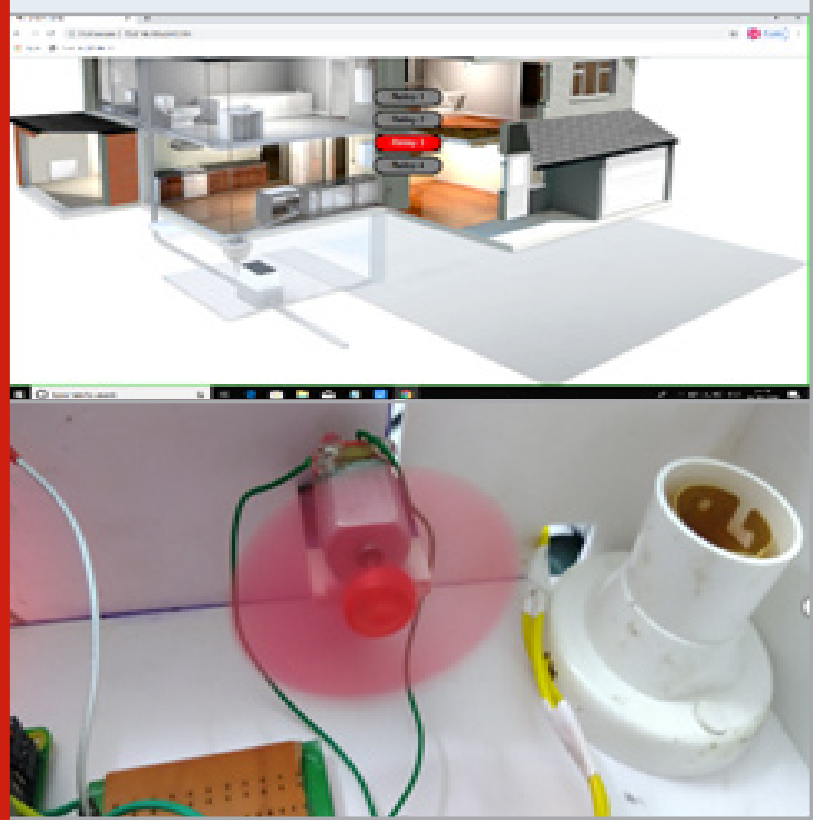

Figure 4.5: Fan and load is controlled by the relay button $1 \mathrm{ft} 3$ at a time from web page

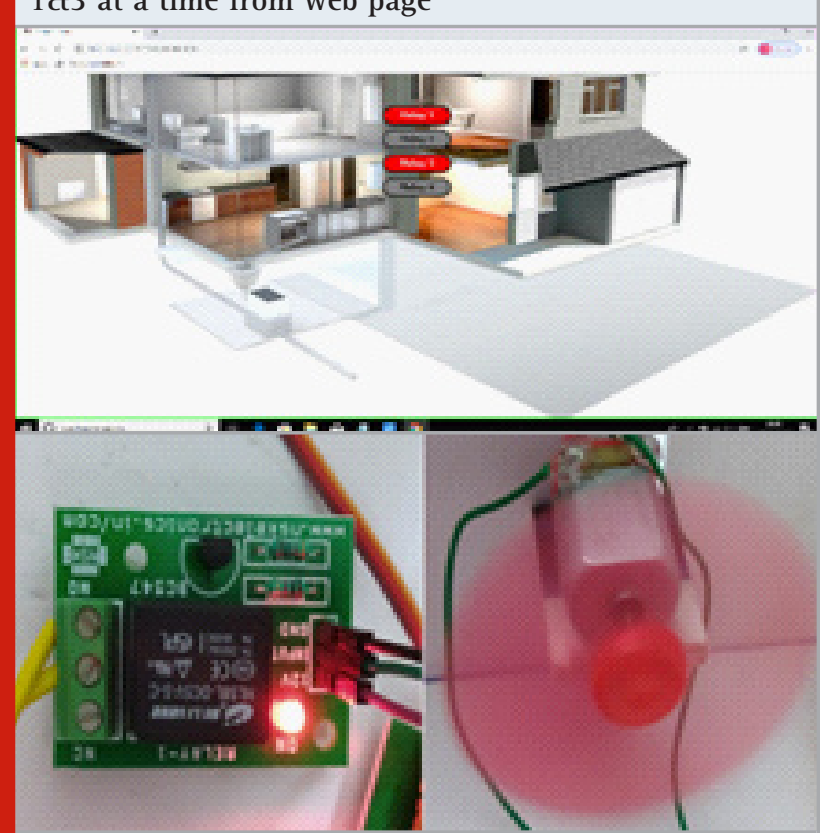

Figure 4.6: Detection of PIR sensor Ct camera opens

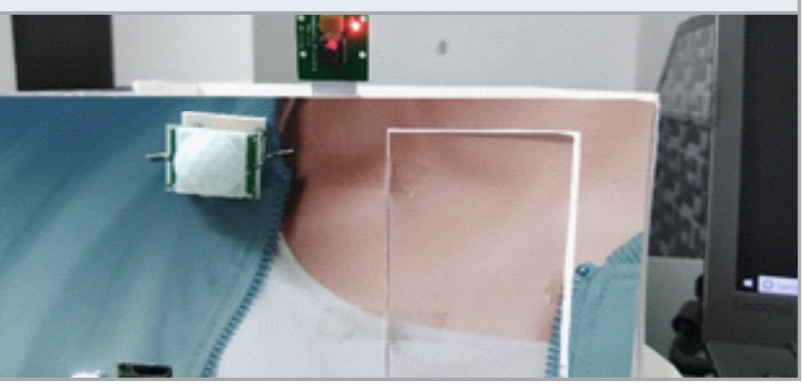

Figure 4.7: Authorised person with matching Id

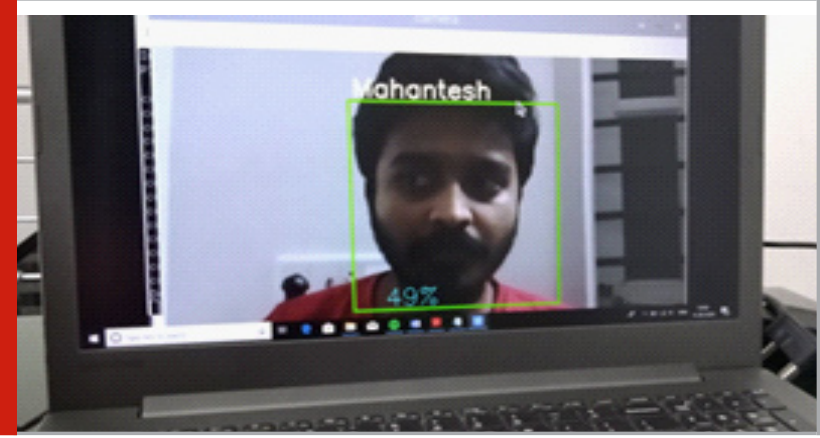

Figure 4.8: Door opens for the authorised person

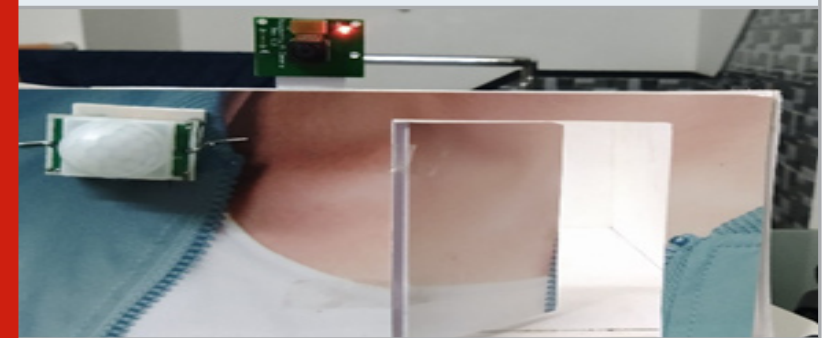


Figure 4.9: Unauthorised person image is sent to mentioned email-Id
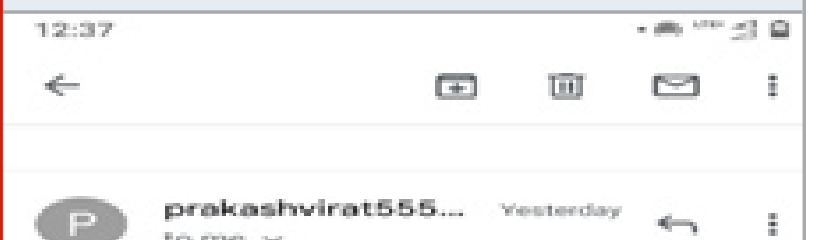

tome s

$\frac{7}{2}$

Unknown Personunknown Person

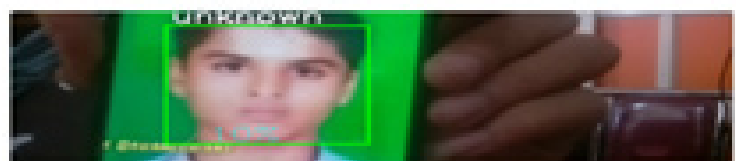

$\underline{1.4}$

noname
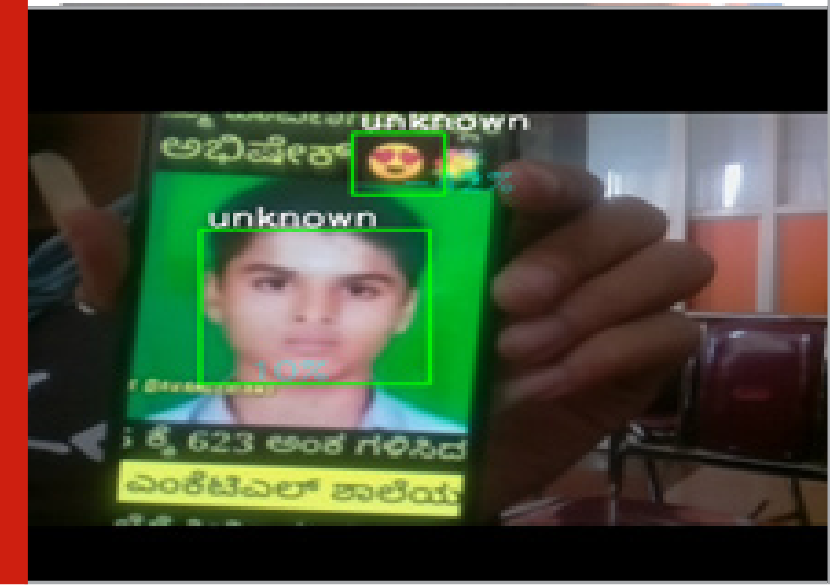

Figure 4.10: Comparison of existing and proposed model

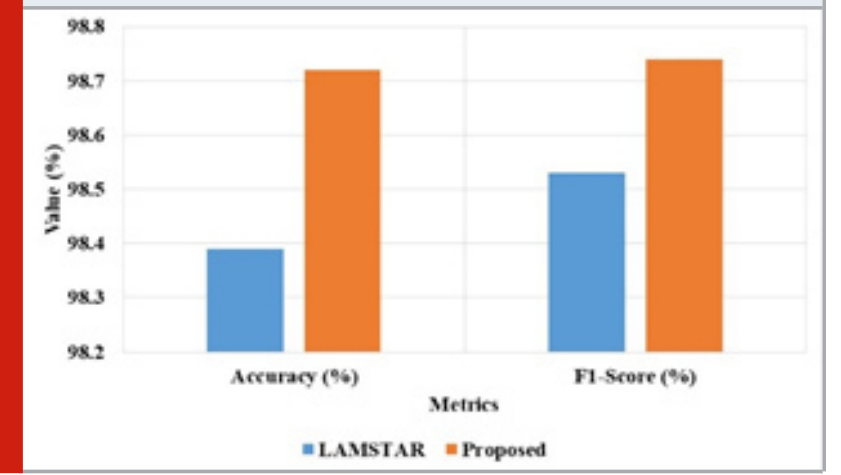

The proposed model is compared with existing face recognition mode of LAMSTAR (Pawar et al. 2018) in IoT, as shown in Table 4.1 and Figure 4.10. The analysis shows that the proposed model has the higher performance compared to existing LAMSTAR (Pawar et al. 2018) model.

\section{CONCLUSION}

Home Automation is the most trending technology currently. This form of technology includes a lot of prospects from a lot of topics or subject assembled
Table 4.1. Comparison of the existing and proposed model

\begin{tabular}{|l|c|c|}
\hline Methods & Accuracy (\%) & F1-Score (\%) \\
\hline LAMSTAR [16] & 98.39 & 98.53 \\
\hline Proposed & 98.72 & 98.74 \\
\hline
\end{tabular}

together. Home automation here has almost brought a evolutionary change in handling or operating home appliances and equipments and made it easy and convenient to use home appliances. A lot of home appliances can be controlled through home automation such as lights, fans, TV, air conditioners, fridge etc. Home automation helps in operating these appliances from far away.

Proposed scheme an effort has been made to build a home automation system with the main virtue of Face Recognization. Face Recognization here has been done with the help of a Raspberry Pi camera which was used to take pictures of the faces and store it in the database once motion is detected by the PIR sensor; camera opens automatically and checks for the authorised person face for the given time. If person face is matched with the previously stored datasets with unique ids then the door opens.

The door only opens after a successful recognization process, when the face is stored previously in the database and that image has got the access to open the door then it will work otherwise it won't open. Otherwise camera captures the image of a person and sends it to the mentioned or desired Email Id.

On Open CV, which is a library feature in the Python language and an open source, all the acknowledgement was done. OpenCV is a library of open source computer vision applications. The library has a lot of streamlined algorithms, including face detection and recognition, which can be used in many IOT-related sectors. LBPH face recognizer, as the project libraries use the HAAR classifier. If the matching index after recognition is more than 25 percent, face recognization is good.

\section{REFERENCES}

Abdel-Basset, M., Manogaran, G., Gamal, A. and Chang, V., 2019. A novel intelligent medical decision support model based on soft computing and IoT. IEEE Internet of Things Journal, 7(5), pp.4160-4170.

Arun, M., Baraneetharan, E., Kanchana, A. and Prabu, S., 2020. Detection and monitoring of the asymptotic COVID-19 patients using IoT devices and sensors. International Journal of Pervasive Computing and Communications.

Casado-Vara, R., Novais, P., Gil, A.B., Prieto, J. and Corchado, J.M., 2019. Distributed continuous-time fault estimation control for multiple devices in IoT networks. 
IEEE Access, 7, pp.11972-11984.

Dautov, R., Distefano, S., Bruneo, D., Longo, F., Merlino, G., Puliafito, A. and Buyya, R., 2018. Metropolitan intelligent surveillance systems for urban areas by harnessing IoT and edge computing paradigms. Software: Practice and experience, 48(8), pp.14751492.

Gandhi, U.D., Kumar, P.M., Varatharajan, R., Manogaran, G., Sundarasekar, R. and Kadu, S., 2018. HIoTPOT: surveillance on IoT devices against recent threats. Wireless personal communications, 103(2), pp.11791194.

Guo, H., Liu, J., Zhang, J., Sun, W. and Kato, N., 2018. Mobile-edge computation offloading for ultradense IoT networks. IEEE Internet of Things Journal, 5(6), pp.4977-4988.

Hasan, M., Islam, M.M., Zarif, M.I.I. and Hashem, M.M.A., 2019. Attack and anomaly detection in IoT sensors in IoT sites using machine learning approaches. Internet of Things, 7, p.100059.

Khan, P.W., Byun, Y.C. and Park, N., 2020. A data verification system for CCTV surveillance cameras using blockchain technology in smart cities. Electronics, 9(3), p.484.

Medapati, P.K., Tejo Murthy, P.H.S. and Sridhar, K.P., 2020. LAMSTAR: For IoT-based face recognition system to manage the safety factor in smart cities. Transactions on Emerging Telecommunications Technologies, 31(12), p.e3843.

Memos, V.A., Psannis, K.E., Ishibashi, Y., Kim, B.G. and Gupta, B.B., 2018. An efficient algorithm for mediabased surveillance system (EAMSuS) in IoT smart city framework. Future Generation Computer Systems, 83, pp.619-628.

Patchava, V., Kandala, H.B. and Babu, P.R., 2015, December. A smart home automation technique with raspberry pi using iot. In 2015 International conference on smart sensors and systems (IC-SSS) (pp. 1-4). IEEE.

Pawar, S., Kithani, V., Ahuja, S. and Sahu, S., 2018, August. Smart Home Security Using IoT and Face Recognition. In 2018 Fourth International Conference on Computing Communication Control and Automation (ICCUBEA) (pp. 1-6). IEEE.

Quadri, S.A.I. and Sathish, P., 2017, June. IoT based home automation and surveillance system. In 2017 International Conference on Intelligent Computing and Control Systems (ICICCS) (pp. 861-866). IEEE.

ShariqSuhail, M., ViswanathaReddy, G., Rambabu, G., DharmaSavarni, C.V.R. and Mittal, V.K., 2016, September. Multi-functional secured smart home. In 2016 International Conference on Advances in Computing, Communications and Informatics (ICACCI) (pp. 2629-2634). IEEE.

Shin, M., Paik, W., Kim, B. and Hwang, S., 2019. An IoT platform with monitoring robot applying CNN-based context-aware learning. Sensors, 19(11), p.2525.

Wati, D.A.R. and Abadianto, D., 2017, November. Design of face detection and recognition system for smart home security application. In 2017 2nd International conferences on Information Technology, Information Systems and Electrical Engineering (ICITISEE) (pp. 342347). IEEE.

Xu, X., He, C., Xu, Z., Qi, L., Wan, S. and Bhuiyan, M.Z.A., 2019. Joint optimization of offloading utility and privacy for edge computing enabled IoT. IEEE Internet of Things Journal, 7(4), pp.2622-2629. 